\title{
Exploring the Usage of 3D Virtual Worlds and Kinect Interaction in Exergames with Elderly
}

\author{
Hugo Paredes ${ }^{1,3}$, Fernando Cassola ${ }^{1,3}$, Leonel Morgado ${ }^{2,3}$, \\ Fausto de Carvalho ${ }^{4}$, Silvia Ala $^{1}$, Francisco Cardoso ${ }^{1}$, \\ Benjamim Fonseca ${ }^{1,3}$, and Paulo Martins ${ }^{1,3}$ \\ 1 UTAD - Universidade de Trás-os-Montes e Alto Douro, Quinta de Prados, \\ Vila Real, Portugal \\ \{hparedes, fcardoso, benjaf, pmartins\}@utad.pt \\ ${ }^{2}$ UAb - Universidade Aberta Av. Dr. Jacques Delors, Porto Salvo, Oeiras, Portugal \\ leonel.morgado@uab.pt \\ 3 INESC TEC - INESC Technology and Science (formerly INESC Porto), \\ Rua Dr. Roberto Frias 378 - Porto, Portugal \\ fernando.c.marques@inescporto.pt \\ 4 PT InS - PT Inovação e Sistemas, Rua Eng. José Ferreira Pinto Basto - Aveiro, \\ Portugal \\ cfausto@telecom.pt
}

\begin{abstract}
The combination of the potentialities of the interactive technologies, like exergames and the emerging motion capture devices with the ability of $3 \mathrm{D}$ virtual worlds for socialisation and context, can produce a platform to promote the physical activity of its users, which leverages its potential. The OnlineGym is an exploratory project based on an online $3 \mathrm{D}$ virtual worlds platform that allows users to interact with the system through the use of a motion capture device. This paper discusses the chosen technological approaches and the preliminary results of the experiments performed with users.
\end{abstract}

Keywords: Active Ageing, Virtual Worlds, Physical Activity, OnlineGym.

\section{Introduction}

Seniors commonly lack physical fitness and often suffer from severe mobility problems. Despite the lack of motivation for physical activities $[3$, general guidelines suggest exercising programs for elderly to strengthen muscles, balance, endurance and mobility 10 .

The OnlineGym is an exploratory project based on an online 3D virtual worlds platform that allows users to interact with the system through the use of a motion capture device such as Microsoft Kinect. It aims to create an "online gymnasium": a virtual three-dimensional space where different users, physically apart, attend a shared workout session coached by a monitor, all of them connected over 
the Internet and represented by avatars directly animated by the movement captured on the Kinect devices connected to each personal computer. This scenario provides the experience of a joint participation in a group gymnastics session. The project is targeted at the elderly, who may not be able to participate in regular training sessions outside their homes, when living in lower-density areas or complex urban neighbourhoods, since that may require expensive or troublesome travel. It is hard to motivate oneself to exercise regularly alone at home 3 , so exergames may help to motivate the elderly to exercise more, providing social interactions with online technologies 34 .

This paper discusses the approaches that have been chosen to fulfil the project requirements and the preliminary results of the experiments performed with users. In the following section the background for the project is presented followed by a brief presentation of the technological platform. The preliminary experiments and results are discussed in section 4 and the paper end with some final remarks.

\section{Background}

In recent years, with the development of virtual reality and tracking technologies a new generation of games has risen: exergames. Such games focus on the development/leverage of the sensory and motor skills of the players, thanks to the possibility of perceptual emulation[5]. Research shows that exergames can have a positive effect on users health[10. However, the motivation of the elderly for the games is closely related to the social factor 3 . Despite the proven potential at the interaction level, impact and visibility levels of exergames, some studies underline the need to strength the social aspects 6. The elderly have been exposed to numerous technological changes, having telecommunications and Web brought a shift in their everyday life 11. This change of interaction, socialisation and communication 2 facilitated connections with family and friends [1 keeping them socially connected and informed $\underline{8}$.

Virtual worlds environments are digital representations of real or imaginary scenarios that intend to simulate reality or compliment it and enable multiple users to be present and communicate with each other 7]. These 3D platforms, which are multi-user, collaborative, and shared virtual environments, can bring the users' experiences to a higher level and bring the being their presence 9 . Morgado et al. [7 put forward the possibility of using these online multiuser spaces becoming the place of real activities, not just simulated ones.

\section{OnlineGym Platform}

The OnlineGym platform is a prototype of an innovative online 3D service, which aims to contribute to the physical and mental well-being of the elderly, based on fitness group activities, where the motor skills and socialisation have essential roles. Its implementation is based on a client-server architecture through which current motion capture technologies allow the elderly to participate in group fitness sessions. 
The objectives of the OnlineGym technological platform are: G1) representation of the avatars 3D movements (puppeteering); G2) remote transmission of animation control for visualisation of the entire group of gymnasts in a shared space; and G3) synchronisation of movements performed by the various participants in order to minimize the effects of communication latency. The achievement of this objectives led to the definition of an architecture with two servers: virtual worlds server and a movements' server. Three platforms that allow interaction within 3D virtual worlds using Kinect-based mocap technology were analysed: FAAST, RINIONS, NUILIB. The RINIONS platform was selected due to its capacity of reproduction of free movements, which ensures the required flexibility to the development. Achieving the objectives G2 and G3 went through adapting the RINIONS platform, jointly with its authors, to support the transmission of gestures. Management modules were also included to support the synchronisation of gestures transmission and minimise the effects of communication latency.

The OnlineGym platform was used to develop a set of experiments with users in order to technically evaluate the implemented features and impacts on the acceptance and adequacy of prototype for the users.

\section{User Experiments}

Experiments for technical evaluation of the OnlineGym platform were held during the development of the prototype and were made locally between the development team of the system, as well as remotely, between the various institutions involved in the project.

A pre-test with a restricted set of users was conducted to understand the degree of acceptance and suitability of the prototype. The results can not be generalised to the general population. However the given indicative data provided a more descriptive and qualitative guidance for future developments. The test was conducted with four participants and a personal trainer, all of which were accompanied by a staff member. Participants voluntarily joined the study, by invitation, and were separated geographically.

The pre-test provided important information regarding the acceptance of the OnlineGym and warned of some aspects that must be taken into account in future development, among which we highlight: the difficulty of the elderly to follow the guidelines given by personal trainer because of the speed of execution of instructions and exercises; the sound quality; the need for increased interactions and use of praise; and the accuracy of the system and the possibility of correcting the positions of the elderly in exercises. Despite the setbacks noted, participants revealed spontaneous readiness to return to use the platform which allows understanding their satisfaction with the experience carried out.

\section{Final Remarks}

In this paper a platform for exergames, based on virtual worlds technology, which allows natural interactions using the Microsoft Kinect motion sensor was presented. The evaluation carried out in a pre-test with users revealed the interest 
and importance of this project, given the positive evaluation of the participants, who were keen to re-use the system.

The preliminary study reveals the need for its continuity and suggests a longitudinal research, with a duration of at least three months, including a group of elderly, physical exercises, interviews and participatory observations, a significant sample of the population, extending the review to effects produced in the aspects of physical and psychological well-being.

Acknowledgments. This work is funded by Portugal Telecom Inovação under the Innovation Plan of the PT Group, and by FEDER funds through the Programa Operacional Fatores de Competitividade COMPETE and National Funds through FCT - Foundation for Science and Technology under the project FCOMP - 01-0124-FEDER-022701.

\section{References}

1. Ariyachandra, T., Crable, E., Brodzinski, J.: Seniors' perceptions of the web and social networking. Issues in Information Systems 10(2), 324-332 (2009)

2. Bachrach, Y., Kosinski, M., Graepel, T., Kohli, P., Stillwell, D.: Personality and patterns of facebook usage. In: Proceedings of the 3rd Annual ACM Web Science Conference, pp. 24-32. ACM (2012)

3. Brox, E., Luque, L., Evertsen, G., Hernandez, J.: Exergames for elderly: Social exergames to persuade seniors to increase physical activity. In: 2011 5th International Conference on Pervasive Computing Technologies for Healthcare (PervasiveHealth), pp. 546-549 (May 2011)

4. Gerling, K., Livingston, I., Nacke, L., Mandryk, R.: Full-body motion-based game interaction for older adults. In: Proceedings of the SIGCHI Conference on Human Factors in Computing Systems, CHI 2012, pp. 1873-1882. ACM, New York (2012)

5. von Bruhn Hinné, T., Keates, S.: Using Motion-Sensing Remote Controls with Older Adults. In: Stephanidis, C. (ed.) Universal Access in HCI, Part II, HCII 2011. LNCS, vol. 6766, pp. 166-175. Springer, Heidelberg (2011)

6. Marston, H.R., Greenlay, S., van Hoof, J.: Understanding the nintendo wii and microsoft kinect consoles in long-term care facilities. Technology and Disability 25(2), 77-85 (2013)

7. Morgado, L., Varajao, J., Coelho, D., Rodrigues, C., Sancin, C., Castello, V.: The attributes and advantages of virtual worlds for real world training. The Journal of Virtual Worlds and Education 1(1), 15-36 (2010)

8. Pettijohn, T.F., LaPiene, K.E., Horting, A.L.: Relationships between facebook intensity, friendship contingent self-esteem, and personality in US college students. Cyberpsychology 6(1) (2012)

9. Schroeder, R.: Defining virtual worlds and virtual environments. Journal For Virtual Worlds Research 1(1) (2008)

10. Waerstad, M., Omholt, K.A.: Exercise Games for Elderly People: Identifying important aspects, specifying system requirements and designing a concept. Institutt for telematikk (2013) 